\title{
Crystal Structure of a Major Outer Membrane Protein from Thermus thermophilus HB27
}

\author{
Alexander Brosig, Jutta Nesper*, Winfried Boos, Wolfram Welte \\ and Kay Diederichs*
}

Department of Biology,

University of Konstanz,

Universitätsstrasse 10, 78457

Konstanz, Germany

Edited by R. Huber
The thermophilic eubacterium Thermus thermophilus belongs to one of the oldest branches of evolution and has a multilayered cell envelope that differs from that of modern Gram-negative bacteria. Its outer membrane contains integral outer membrane proteins (OMPs), of which only a few are characterized. Tto $A$, a new $\beta$-barrel OMP, was identified by searching the genome sequence of strain HB27 for the presence of a C-terminal signature sequence. The structure of TtoA was determined to a resolution of $2.8 \AA$, representing the first crystal structure of an OMP from a thermophilic bacterium. TtoA consists of an eight-stranded $\beta$-barrel with a large extracellular part to which a divalent cation is bound. A five-stranded extracellular $\beta$-sheet protrudes out of the membrane-embedded transmembrane barrel and is stabilized by a disulfide bridge. The edge of this $\beta$-sheet forms crystal contacts that could mimic interactions with other proteins. In modern Gram-negative bacteria, the C-terminal signature sequence of OMPs is required for binding to an Omp85 family protein as a prerequisite for its assembly. We present hints that a similar assembly pathway exists in T. thermophilus by an in vitro binding assay, where unfolded TtoA binds to the Thermus Omp85 family protein TtOmp85, while a mutant without the signature sequence does not.

Keywords: outer membrane biogenesis; thermophile; T. thermophilus; TTC0834; TTC1475

\section{Introduction}

Thermus thermophilus, which was originally isolated from a hot spring, is a thermophilic eubacterium with an optimal growth temperature of $70^{\circ} \mathrm{C}$. It most likely represents an evolutionary intermediate between Gram-positive bacteria and modern Gram-negative bacteria, ${ }^{1}$ as is reflected also by an unusual cell envelope. A unique peptidoglycan ${ }^{2}$ to which a secondary cell wall polysaccharide is covalently linked ${ }^{3}$ encloses the inner membrane. This cell wall is surrounded by an outer membrane (OM) and a surface layer (S-layer). The S-layer protein is regularly

*Corresponding authors. E-mail addresses: Kay.Diederichs@uni-konstanz.de; Jutta.Nesper@uni-konstanz.de.

Abbreviations used: OM, outer membrane; OMP, outer membrane protein; S-layer, surface layer; SIRAS, single isomorphous replacement with anomalous scattering; C8E4, n-octyltetraoxyethylene; PBS, phosphate-buffered saline. arrayed ${ }^{4}$ but is unusual since it shares some structural characteristics with porins ${ }^{4,5}$ and connects the OM/Slayer to the secondary cell wall polysaccharide. ${ }^{3}$ The OM contains specific glycolipids and phosphoglycolipids but not lipopolysaccharide. 6,7

Integral $\beta$-barrel proteins are the most abundant proteins in the OM of modern Gram-negative bacteria. They function as channels for nutrients (e.g., nonspecific porins, specific channels or active transporters), exporters for proteins or hydrophobic toxic substances, enzymes, adhesion molecules, assembly factors and anchors to the cell wall. Several structures of transmembrane $\beta$-barrel OM proteins (OMPs) containing invariably all-next-neighbor antiparallel $\beta$-strands with a number of strands within the barrel ranging between 8 and 22 have been solved.

So far, no structure of an integral OMP from $T$. thermophilus is known. Moreover, only little information about OMPs is available. In black lipid bilayer experiments using OM preparations from strain HB8, a 185-kDa protein was identified as a putative porin that formed unusually large pores. ${ }^{8}$ For strain HB27, Rumszauer et al. described OMPs involved in pilus 
biogenesis. ${ }^{9}$ We previously characterized TtOmp85, an Omp 85 family protein of $\mathrm{HB} 27$, as a ring-like, poreforming and primarily monomeric OMP. ${ }^{10}$ Apart from TtOmp85, no further putative $\beta$-barrel OMP was predicted on the basis of sequence homology in the genome sequences of HB27 and HB8. ${ }^{11,12}$ Prediction of $\beta$-barrel OMPs from the primary sequence is still difficult because of the presence of short transmembrane stretches. Furthermore, the interior of the barrel is not always hydrophilic; therefore, searching for alternating polar and nonpolar residues is not promising. ${ }^{13}$

In modern Gram-negative bacteria, all $\beta$-barrel OMPs contain an N-terminal signal sequence for translocation across the inner membrane by the secretion machinery, and a related pathway is present in T. termophilus. ${ }^{14}$ In addition, OMPs from modern Gram-negative bacteria with the $\beta$-barrel part at the $C$-terminus have a signature sequence corresponding to the last $\beta$-strand. This sequence shows Phe or Trp at the C-terminal position and hydrophobic residues at positions 3, 5, 7 and 9 as counted from the Cterminus. ${ }^{15}$ Prior to insertion and folding (collectively called "assembly"), the nonnative processed OMPs interact via this signature sequence with an Omp85 family protein. ${ }^{16}$ Such a C-terminal signature sequence is also present in the Omp85 family protein TtOmp85, indicating that a related pathway for assembly may exist in T. thermophilus and that TtOmp85 itself is the acceptor. Therefore, the Cterminal signature sequence was assumed to be suitable to identify together with the $\mathrm{N}$-terminal signal sequence new $\beta$-barrel OMPs for structural analysis and for studying the assembly process.

We used bioinformatics and searched the genome of HB27 sequentially for proteins with three features: (i) a C-terminal signature sequence, (ii) an $\mathrm{N}$-terminal signal sequence and (iii) $\beta$-strands. Two new proteins met these criteria: TTC1475 and TTC0834, named TtoA. Using an OM preparation, we identified one major OMP by mass spectrometric analysis as TtoA. Therefore, we characterized TtoA and solved its structure. TtoA shows a typical antiparallel $\beta$-barrel structure with eight strands and a complex extracellular part. In addition, a first hint that the C-terminal signature sequence of TtoA is involved in its biogenesis, since this motif was necessary for efficient binding of unfolded TtoA to TtOmp85 in in vitro binding assays, was found.

\section{Results}

\section{TtoA is an OMP}

The genome sequence of T. thermophilus HB27 was searched for proteins containing the $\mathrm{C}$-terminal signature sequence. ${ }^{15}$ All these proteins were checked for a predicted $\mathrm{N}$-terminal signal sequence and predicted $\beta$-strands. This method led to the identification of three potential OMPs having a $\beta$-barrel structure: TtOmp85 (TTC0193); TTC0834, which we named TtoA (T. thermophilus OMP A) herein; and TTC1475. The hypothetical protein TTC1475 is predicted to have a molecular mass of $15.5 \mathrm{kDa}$ (mature sequence) and $10 \beta$-strands. TTC1475 is encoded in an operon together with TTC1476, which is predicted to be an inner membrane transporter containing six transmembrane helices.

In addition, OMP preparations of strain HB27 were separated by SDS-PAGE (sodium dodecyl sulfate polyacrylamide gel electrophoresis), and prominent bands were identified by mass spectrometric analysis. The two major proteins correspond to the S-layer protein and TtoA (Fig. 1a). Since TtoA was identified by both methods, bioinformatics and

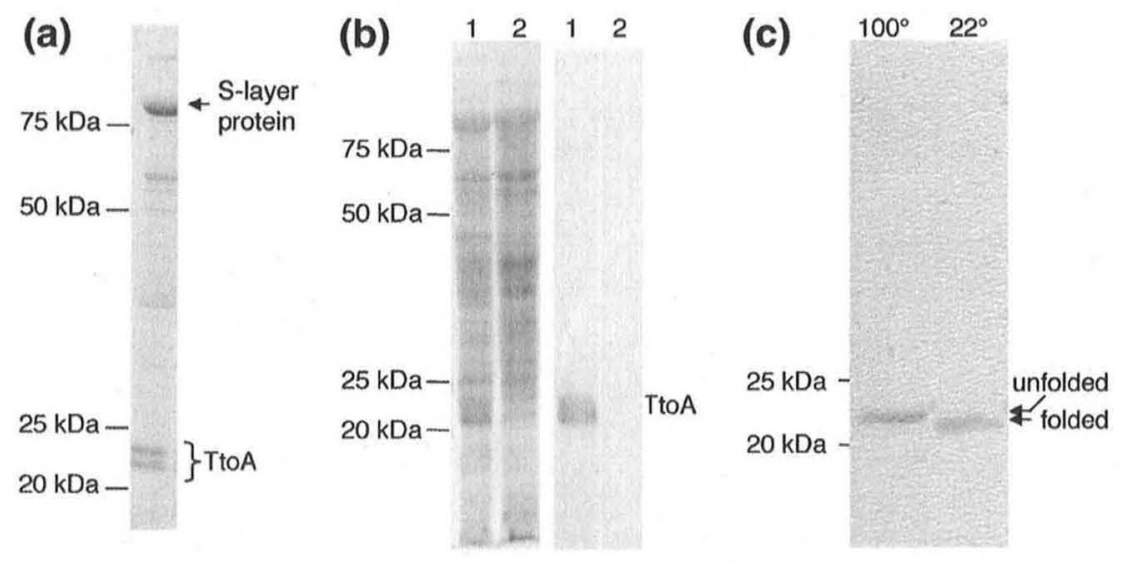

Fig. 1. TtoA is an HB27-specific major OM $\beta$-barrel protein. (a) Identification of OMPs from T. thermophilus HB27. OMPs were separated by SDS-PAGE, and the gel was stained with Coomassie G250. The named proteins were identified by mass spectrometric analysis. TtoA appears as a double band because it was not completely denatured [compare with (c)]. (b) Physiological expression of TtoA in HB27 and HB8. Whole-cell extracts were separated by SDS-PAGE. The left pair of lanes shows a Coomassie-stained polyacrylamide gel. The right pair of lanes shows an immunoblot of the same extract. The blot was probed with anti-TtoA antiserum. Lane 1, whole-cell extract of HB27; lane 2, that of HB8. (c) Purified TtoA showing a heat-modifiable phenotype. Shown is a Coomassie-stained polyacrylamide gel. Upon denaturation by heating $\left(100{ }^{\circ} \mathrm{C}\right)$, Tto $\mathrm{A}$ runs slower than the protein loaded without heating $\left(22^{\circ} \mathrm{C}\right)$. 
biochemistry, we focused on this protein. TtoA is annotated as a hypothetical protein and homology exists only with unknown proteins from Thermus aquaticus, Deinococcus radiodurans and Deinococcus geothermalis. Interestingly, TtoA was not found in the genome sequence of T. thermophilus HB8, and it was also not detectable in our HB8 strain in Western blot analysis (Fig. 1b).

TtoA was purified from OM preparations of HB27. When analyzed by SDS-PAGE, it showed behavior typical for monomeric OM $\beta$-barrel proteins: ${ }^{17}$ Upon denaturation by heating for $20 \mathrm{~min}$ at $100{ }^{\circ} \mathrm{C}$ in SDS-containing buffer, TtoA migrated slower than TtoA, not heated before loading (Fig. 1c).

\section{Structure solution of TtoA}

Recently, we reported the homologous expression of TtoA with an $\mathrm{N}$-terminal His tag in the OM of $T$. thermophilus HB8, the crystallization of His-TtoA in $0.2 \mathrm{M}$ sodium malonate and $44 \%$ methylpentanediol and the diffraction pattern of those crystals. ${ }^{18}$ The crystals were soaked in $\mathrm{K}_{2} \mathrm{PtCl}_{4}$, and the data set collected at a synchrotron was used for phase

Table 1. Summary of data collection and refinement statistics

\begin{tabular}{|c|c|c|}
\hline & Native $(2.8 \AA)$ & $\mathrm{K}_{2} \mathrm{PtCl}_{4}$ \\
\hline \multicolumn{3}{|l|}{ Data set statistics } \\
\hline Unit cell parameters & $\begin{array}{c}a=b=166.3 \AA \\
c=97.7 \AA\end{array}$ & $\begin{array}{c}a=b=166.2 \AA, \\
c=97.8 \AA\end{array}$ \\
\hline & $\begin{array}{c}\alpha=\beta=90^{\circ} \\
\gamma=120^{\circ}\end{array}$ & $\begin{array}{c}\alpha=\beta=90^{\circ} \\
\gamma=120^{\circ}\end{array}$ \\
\hline Space group & $P 3,21$ & $P 3,21$ \\
\hline Resolution range $(\AA)$ & $40-2.8(2.97-2.8)$ & $40-3.5(3.7-3.5)$ \\
\hline$\lambda(\AA)$ & 1.0162 & 0.9252 \\
\hline Oscillation angle $\left(^{\circ}\right)$ & 0.5 & 0.5 \\
\hline No. of unique reflections & $38,811(6021)$ & $37,705(5763)$ \\
\hline No. of observed reflections & $381,009(60,864)$ & $341,205(27,964)$ \\
\hline Completeness (\%) & $99.5(97.2)$ & $98.8(93.4)$ \\
\hline Mean $I / \sigma(I)$ & $19.59(1.42)$ & $15.96(1.20)$ \\
\hline$R_{\text {meas }}{ }^{\text {a }}(\%)$ & $9.3(172.5)$ & $10.7(130.5)$ \\
\hline$R_{\text {mrgd-F }}{ }^{\mathrm{a}}(\%)$ & $9.7(103.4)$ & $14.8(126.0)$ \\
\hline $\begin{array}{l}\text { SIRAS phasing power } \\
\text { (iso/ano) }\end{array}$ & $0.47 / 0.28$ & \\
\hline $\begin{array}{l}\text { SIRAS FOM after SHARP } \\
\text { (centric/acentric) }\end{array}$ & $0.12 / 0.12$ & \\
\hline \multicolumn{3}{|l|}{ Refinement statistics } \\
\hline $\begin{array}{l}\text { Resolution range }(\AA) \\
\text { (highest bin) }\end{array}$ & $\begin{array}{l}37.13-2.80 \\
(2.87-2.80)\end{array}$ & \\
\hline $\begin{array}{l}\text { Total no. of atoms } \\
\text { (nonhydrogen) }\end{array}$ & 4796 & \\
\hline $\begin{array}{l}R_{\text {work }} / R_{\text {free }}(\%) \\
\text { (highest bin) }\end{array}$ & $\begin{array}{c}19.8 / 23.4 \\
(30.3 / 33.9)\end{array}$ & \\
\hline r.m.s.d. bond lengths $(\AA)$ & 0.013 & \\
\hline r.m.s.d. bond angles $\left({ }^{\circ}\right)$ & 1.65 & \\
\hline Average $B$-factor $\left(\AA^{2}\right)$ & 100.7 & \\
\hline $\begin{array}{l}\text { Residues in regions of } \\
\text { Ramachandran plot (\%) } \\
\text { (most favored/ } \\
\text { additional allowed/ } \\
\text { generously allowed/ } \\
\text { disallowed) }\end{array}$ & $87.9 / 9.3 / 1.2 / 1.6$ & \\
\hline $\begin{array}{l}\text { Values in parentheses in } \mathrm{d} \\
\text { resolution shell. } \\
\text { a As defined by Diederic }\end{array}$ & $\begin{array}{l}\text { a set statistics ar } \\
\text { and Karplus. }^{19}\end{array}$ & for the highest- \\
\hline
\end{tabular}

determination via single isomorphous replacement with anomalous scattering (SIRAS; Table 1). An improved set of crystals diffracted to $2.8 \AA$ (Table 1 ), allowing further improvement of the molecular model. All crystals belonged to space group $P 3_{1} 21$ with three monomers per asymmetric unit and a Matthews coefficient of $V_{M}=5.4 \AA^{3} /$ Da corresponding to a solvent content of $77 \%$. His-TtoA without signal sequence (214 residues) includes six His and two Ala residues inserted at position 2 of the annotated mature protein sequence. No electron density was observed for Gln1 and the hexa-His tag; the final model thus starts with two Ala residues followed by Lys 2 and comprises 207 residues. The refined model contains three TtoA monomers, six C8E4 (n-octyltetraoxyethylene) detergent molecules, three divalent cations and 41 water molecules in the asymmetric unit; eight residues located in the loop between $\beta$-strands 5 and 6 of each monomer remained in disallowed regions of the Ramachandran plot (Table 1).

\section{Overall structure of TtoA}

Tto A resembles the fold of known eight-stranded $\beta$-barrel proteins showing an all-next-neighbor upand-down topology of the strands (Fig. 2a). Its overall size is $\sim 59 \AA$ in height with a cross-sectional diameter of the elliptical barrel ranging from 14 to $18 \AA$. On one side of the barrel, the strands are connected by three shorter loops of different lengths. Considering the general observation that the periplasmic side of OMPs consists of short loops and harbors the chain termini, whereas the extracellular part displays longer loops, ${ }^{20}$ Tto $\mathrm{A}$ is most likely oriented in the membrane with the large nonbarrel part toward the exterior (Fig. 2a; topology shown in Fig. 2b). The top of the extracellular part shows the largest circumference with elliptic diameters of 29 and $36 \AA$.

\section{Transmembrane part}

Tto $A$ is organized as a $\beta$-barrel consisting of eight amphipathic $\beta$-strands with nonpolar side chains pointing toward the membrane and polar side chains facing the lumen of the $\beta$-barrel. It displays two rings of aromatic side chains located at the upper and lower interfaces between hydrophilic and hydrophobic parts of the membrane (Fig. 2a). The planes of these girdles lie perpendicular to the barrel axis, indicating an orientation of the axis normal to the membrane plane. The height of the $\beta$-barrel is typical for OMPs. The barrel has a shear number ${ }^{13}$ of 10 , with the individual $\beta$-strands being tilted by $40^{\circ}$ against the barrel axis. Of 27 residues lining the barrel interior, 17 (63\%) contain side-chain lengths of at least three carbon atoms between the protein main chain and the polar "head." These narrow the accessible inner surface of the barrel significantly and, as a consequence, leave no obvious space for a continuous channel (Fig. 3a). Among these, the hydrophilic side chains form a network of hydrogen bonds and salt bridges. 
(a)

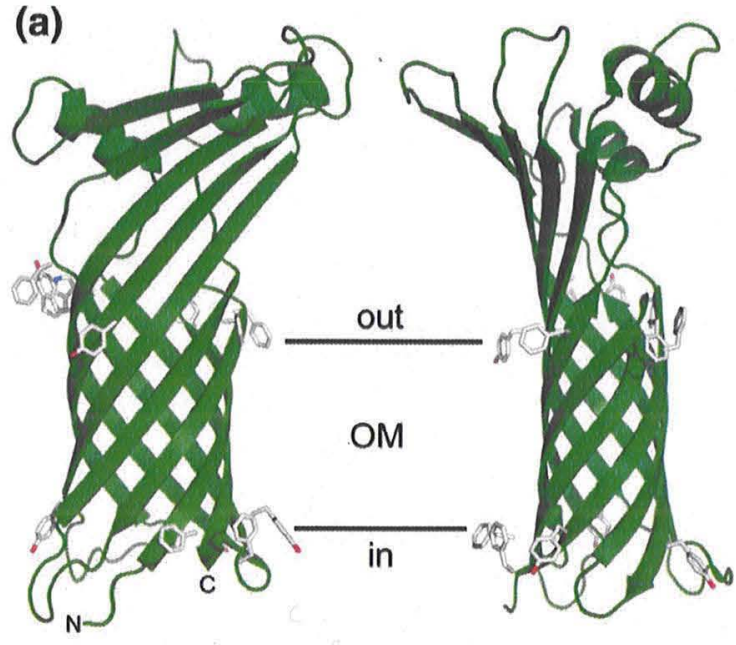

(b)

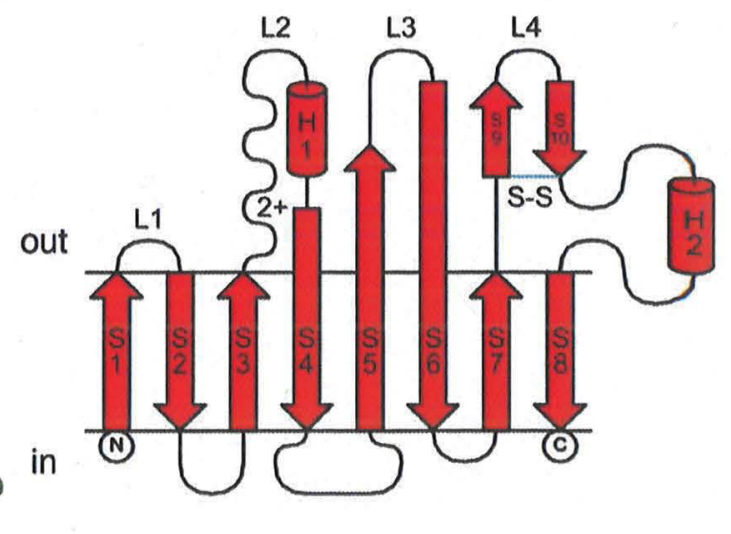

Fig. 2. Overall structure of TtoA. (a) Ribbon representation of the TtoA monomer. The two views differ by a $90^{\circ}$ rotation. The side chains of the two aromatic girdles allow inference of the orientation and position of the barrel in the membrane. (b) Topology of the TtoA fold. The disulfide bridge is shown in cyan, and " $2+$ " marks the position where the divalent cation is bound between extracellular loop 2 and $\beta$-strand 4 . Horizontal lines represent the membrane limits. The $\mathrm{N}$ - and C-termini are indicated.

A remarkable feature can be observed about $7 \AA$ from the periplasmic barrel entrance where the lumen is intersected by residues Glu7 and Arg135 pointing from opposite sides toward each other such that a salt bridge is formed. The remaining space next to that intersection is filled with hydrophobic side chains Val23+Leu82 and Val147, respectively (Fig. $3 b$ and c). As a consequence, this section of the inner surface of the barrel shows both steric and electrostatic occlusions and could serve as a barrier for hydrophilic compounds.

\section{Extracellular part}

The extracellular part of TtoA is formed by extended strands 4,5 and 6 of the $\beta$-barrel and by extracellular loops 2, 3 and 4. It consists of 92 of 207 residues, corresponding to $44 \%$ of the amino acid sequence of a TtoA monomer. The extracellular loops extensively form secondary structure elements: In addition to the three $\beta$-strands protruding out of the $\beta$-barrel, the resulting $\beta$-sheet is extended by two additional antiparallel $\beta$-strands (9 and 10) located in extracellular loop 4 . These attach to strand 6 of the $\beta$ barrel and assemble into an extracellular $\beta$-sheet consisting of five $\beta$-strands in total that is stabilized by a disulfide bridge between strands 9 and 10. In addition, extracellular loops 2 and 4 contribute two $\alpha$ helices, defining together with the $\beta$-sheet the outer rim of the extracellular part (Fig. 4). Taken together, the extracellular part of Tto A forms an asymmetric arrangement with an $\alpha$-helical/coil side opposed to the extended $\beta$-sheet. The surface of that part facing away from the membrane is formed by the upper side of the $\beta$-sheet and the two attached $\alpha$-helices and forms a funnel-like arrangement that narrows toward the upper entrance of the $\beta$-barrel.

Additional spherical electron density was observed in all three monomers between extracellular loop 2 and the extracellular part of strand 4 . This
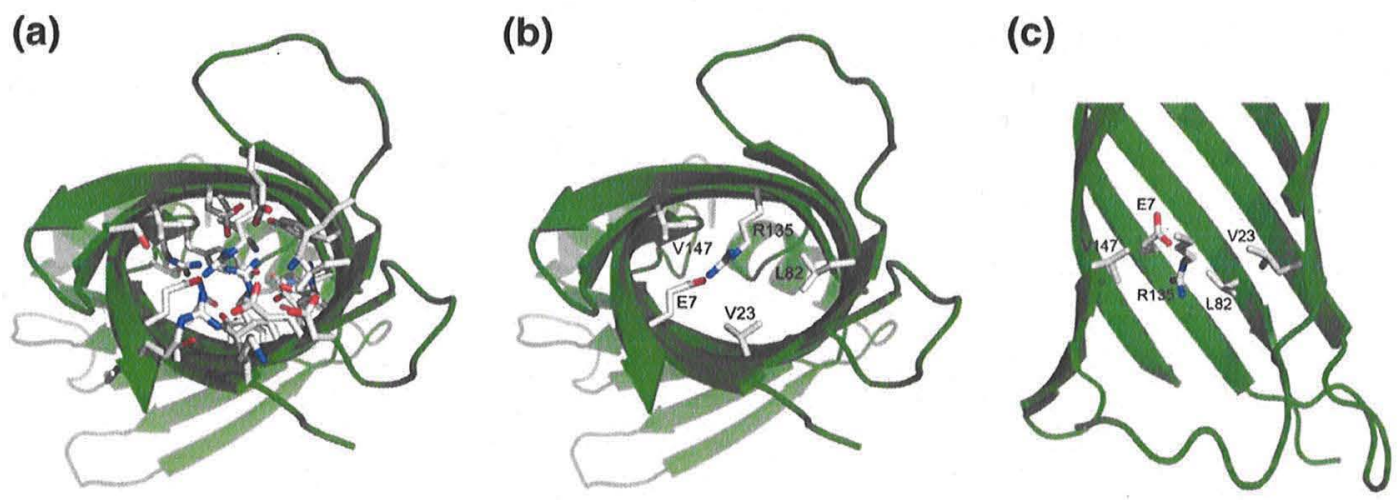

Fig. 3. Lumen of the 3 -barrel with its barrier for hydrophilic compounds. (a) Stick representation of all side chains located in the barrel lumen. (b) Side chains forming the barrier for hydrophilic compounds. (c) Side view of that barrier. Cartoons of $\beta$-sheets blocking the view into the $\beta$-barrel were omitted. 


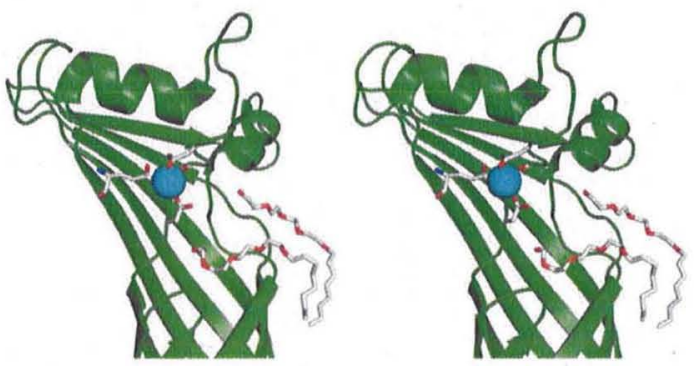

Fig. 4. Stereo representation of the extracellular part. Residues involved in the binding of the divalent cation (cyan) are shown in stick representation. In the center, the possible entrance for hydrophilic compounds into the barrel is occupied by a C8E4 molecule.

density was interpreted as a divalent cation and was modeled in the refined structure as a $\mathrm{Ca}^{2+}$ for several reasons: First, anomalous density was observed at this position for a data set collected from a native His-TtoA crystal at a wavelength of $1.77 \AA$ (data not shown), which is of comparable magnitude with that observed for the two sulfur atoms in the disulfide bridge between Cys159 and Cys177 (expected anomalous signals at $1.77 \AA$ : $f^{\prime \prime}$ calcium $=1.637$ electrons and $f^{\prime \prime}$ sulfur $=0.722$ electrons). Second, the $B$ factors of the calcium ions $\left(111,109\right.$ and $121 \AA^{2}$ for chains $A, B$ and $C$, respectively) are of the same magnitude as those for the coordinating carboxyl oxygens of residues Asp45, Asp49 and Glu70. Third, calcium ions are mostly coordinated in protein structures by aspartate and glutamate residues and altogether show six or seven coordinations. ${ }^{21}$ Calcium was not added during purification or crystallization, suggesting that it could have bound tightly to Tto A in the OM, where $\mathrm{Ca}^{2+}$ is naturally present. ${ }^{22}$

The asymmetry of the extracellular part is also evident in the charge distribution on the surface of TtoA, which exhibits two clusters of negative charges, one around the entrance rim of the funnel, including the upper side of the $\beta$-sheet (Fig. $5 a-c$ ), and another where loops 1, 2 and 4 face one another (Fig. 5a). The inner surface of the funnel close to the upper barrel entrance is characterized by the presence of aromatic and apolar side chains (Ala46, Leu47, Tyr51, Leu53, Trp59, Val62, Phe68, Phe105, Tyr109, Ile123, Phe160, Val176, Val186, Phe189 and Val190) and thus is predominantly hydrophobic (Fig. 5c). It narrows toward the entrance into the barrel, which is hydrophilic.

As expected, the exterior of the barrel is predominantly uncharged (Fig. $5 \mathrm{a}$ and $\mathrm{b}$ ). The charge distribution of the downside of the $\beta$-sheet and the intracellular side is neutral (Fig. 5b).

\section{Crystal contacts}

Crystal contacts are present between transmembrane regions and pairs of symmetry-related extracellular $\beta$-strand 10 . Hydrophobic interactions between the $\beta$-barrels pack TtoA as trimers in the crystal lattice. These form most likely upon crystallization because the barrel axes are tilted with respect to each other, whereas the structure suggests a parallel orientation in the membrane. Moreover, parallel packing of monomers along the barrel axis, as observed in several trimeric porins, is not possible, because the extracellular parts would sterically clash. The arrangement suggests partial displacement of the detergent belt upon crystallization, as has been observed in other OMP crystal lattices. ${ }^{23}$ In contrast, the observed intermolecular $\beta$ sheet formation of $\beta$-strand 10 (Fig. 6) could indeed mimic physiological interactions with other $\beta$-sheetexposing proteins.

\section{Homology of TtoA to other proteins}

The crystal structures of six different eightstranded $\beta$-barrel OMPs from mesophilic organisms have been solved before: OmpA, ${ }^{24} \mathrm{OmpX},{ }^{25}$ $\mathrm{OmpW}^{26}$ and $\mathrm{PagP}^{27}$ from Escherichia coli; PagL from Pseudomonas aeruginosa; ${ }^{28}$ and $\mathrm{NspA}$ from Neisseria meningitidis. ${ }^{29}$ TtoA is unique but shares some features with OmpA, OmpX, OmpW and NspA and less with the two others, PagL and PagP, which are tilted relative to the membrane. Similarly, as observed in OmpA, OmpX and NspA, the interior of the $\beta$-barrel of TtoA contains a network of hydrogen bonds and salt bridges without forming a pore. In addition, TtoA contains a putative barrier for hydrophilic compounds inside the $\beta$-barrel similar to a section observed on the upper side of the $\beta$-barrel of OmpW, with the difference being that the barrier is located within the lower half of the transmembrane part in TtoA. The extracellular part of TtoA displays high structural complexity with a hydrophobic center, similar to the structures of OmpW, NspA and PagP. When looking at the elongated $\beta$-strands protruding out of the barrel part into the extracellular environment, strands 4, 5 and 6 of OmpA, OmpX and TtoA are involved in (a)

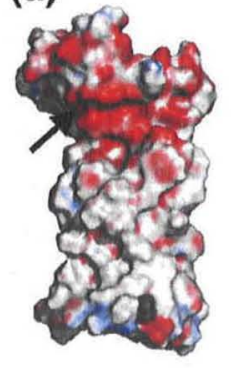

(b)

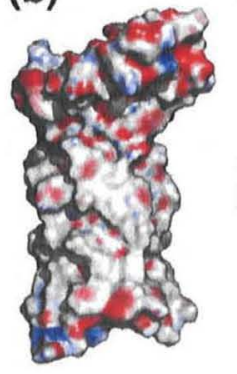

(c)

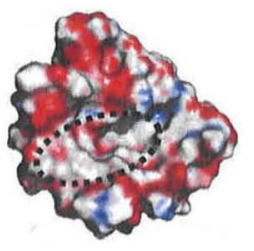

Fig. 5. Charge distribution on the surface of TtoA. Negative charges are shown in red; positive charges, in blue. The color gradient represents the charge density. (a) Side view of TtoA, oriented as in Fig. 4. The divalent cation binds to the prominent negatively charged patch (arrow). (b) View rotated by $180^{\circ}$ compared with (a). (c) Top view of TtoA showing a charged rim around the extracellular part and the apolar patch in the center of the funnel (dashed ellipse). 


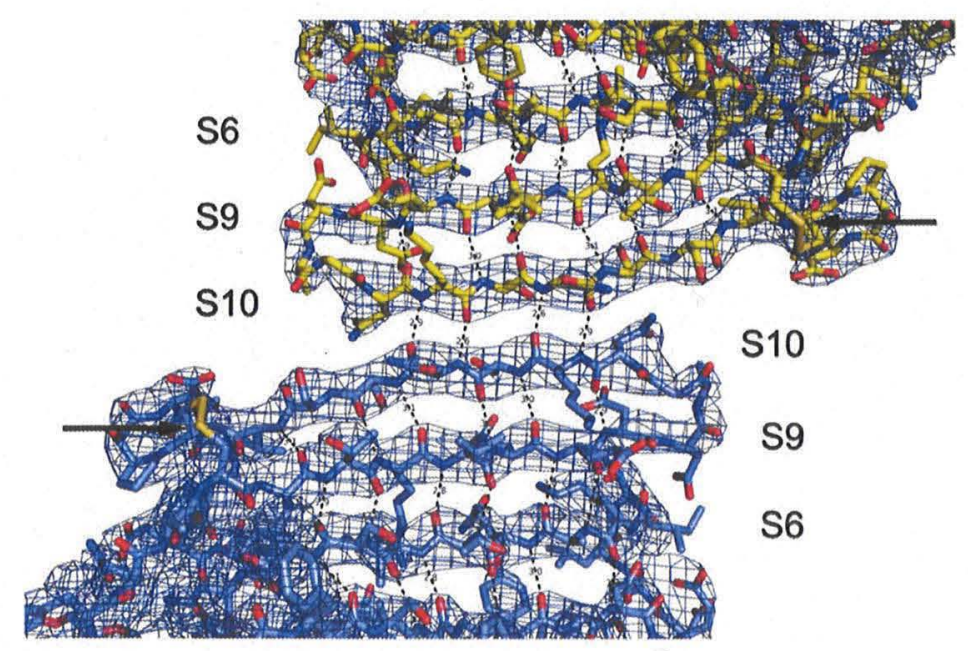

Fig. 6. Crystal contact of putative physiological interest. Shown are crystal contacts between $\beta$ strands 10 of neighboring monomers in the crystal lattice forming an intermolecular $\beta$-sheet. Monomers are shown in blue and yellow. The electron density $\left(2 F_{\mathrm{o}}-F_{\mathrm{c}}\right.$ map) is indicated at a cutoff of $2 \sigma$. The disulfide bridge between strands 9 and 10 is indicated in both molecules by arrows. The strands (S) are numbered according to the topology representation in Fig. 2.

forming extracellular $\beta$-sheets, which contain four $\beta$-strands in OmpA and OmpX and five in TtoA, respectively.

Sequence similarity to TtoA (Fig. 7) is found only in TaqDRAFT_1754 (59\% identity) from T. aquaticus Y51MC23 (optimal growth temperature of $70{ }^{\circ} \mathrm{C}$ ), DR_0972 (32\% identity) from D. radiodurans R1 $\left(30{ }^{\circ} \mathrm{C}\right)$ and Dgeo_0718 (30\% identity) from $D$. geothermalis $\left(47^{\circ} \mathrm{C}\right)$. Residues forming the $\beta$-barrel are well conserved among all proteins, but residues forming the barrier for hydrophilic compounds within the barrel are only present in T. aquaticus. Main differences between the sequences are found in the extracellular loops; therefore, we examined whether the remarkable features of the extracellular part are conserved. Residues forming the hydro-

phobic patch are well conserved in the homolog from T. aquaticus, whereas the residues are mainly exchanged to other hydrophobic amino acids in the homologs from $D$. radiodurans and D. geothermalis. Only the homolog of $D$. geothermalis does not contain the residues involved in the binding of the divalent cation, but it does contain two cysteines that are not found in the homologs of $T$. aquaticus and $D$. radiodurans.

The C-terminal signature sequence of TtoA is necessary for efficient binding to TtOmp85

OMPs of modern Gram-negative bacteria containing a C-terminal signature sequence bind to an Omp85 family protein on the OM before becoming

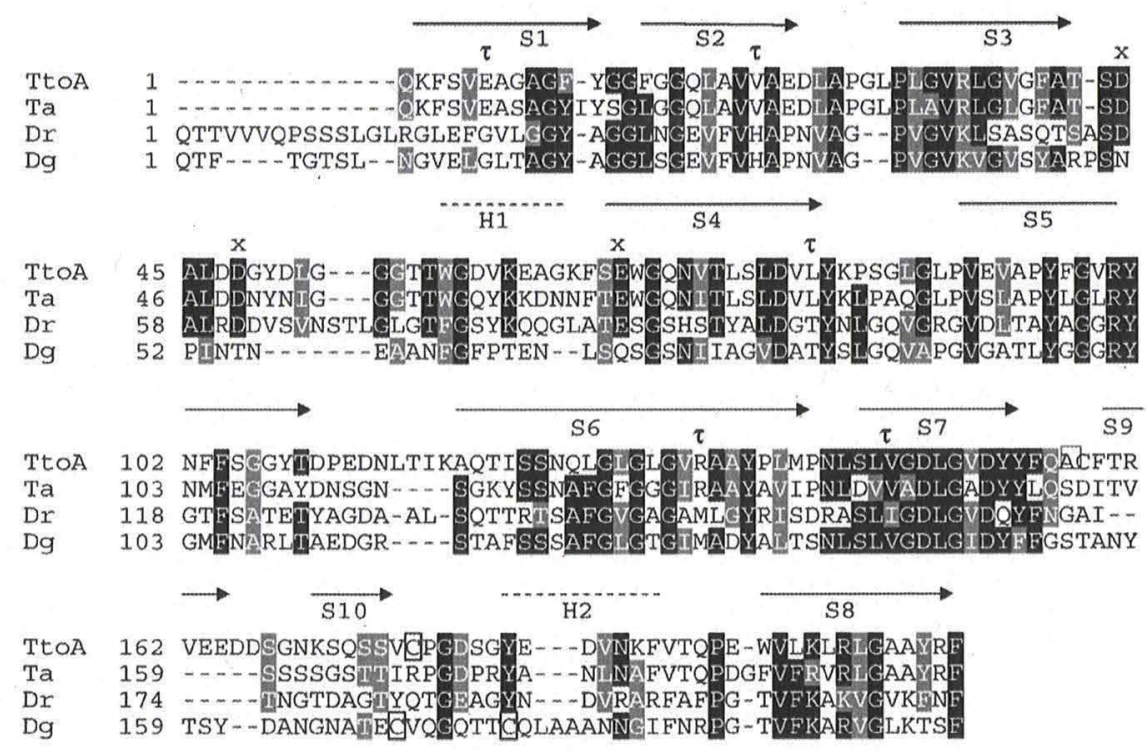

Fig. 7. Alignment of TtoA homologs. Identical residues $(\geq 3)$ are shown in black, whereas conserved residues are shown in gray. " $\tau$ " marks residues involved in the barrier for hydrophilic compounds. " $X$ " presents residues binding the divalent cation. Cysteines are boxed. The secondary structure of TtoA as determined with the program DSSP is indicated at the top. Arrows indicate $\beta$-stands (S1-S10), whereas dotted lines indicate $\alpha$-helices $(\mathrm{H} 1-\mathrm{H} 2)$. Proteins aligned: Tto A from T. thermophilus HB27 (TtoA), TaqDRAFT_1754 from T. aquaticus Y51MC23 (Ta), DR_0972 from D. radiodurans R1 (Dr) and Dgeo_0718 from D. geothermalis (Dg). 


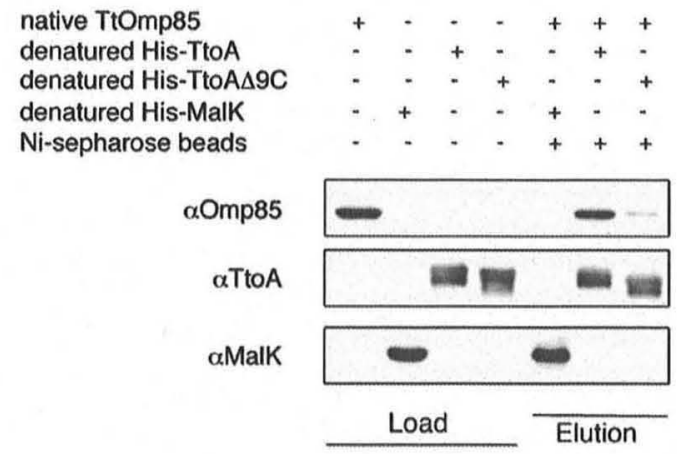

Fig. 8. Pull-down assay showing binding of native TtOm85 only to denatured His-TtoA containing the signature sequence. Urea-denaturated His-TtoA or HisTtoA $\triangle 9 \mathrm{C}$ bound to Ni-Sepharose matrix was incubated with purified TtOmp85, and His-MalK was used in parallel as a control. Bound proteins were analyzed by immunostaining using the indicated primary antibodies $(\alpha)$.

assembled into the OM. ${ }^{16}$ As we identified TtoA via this motif, we investigated in pull-down assays whether TtoA can bind to TtOmp85 and whether this sequence is involved. Tto $A$ and a mutant missing the last 9 aa (Tto $A \Delta 9 \mathrm{C}$ ) were produced with an $\mathrm{N}$-terminal His tag as inclusion bodies in $E$. coli and purified in the presence of urea. We used the purified N-terminally His-tagged cytosolic protein MalK, denatured in urea, as a control protein. The denatured proteins were bound to Ni-Sepharose beads and incubated for $30 \mathrm{~min}$ with purified TtOmp85 solubilized in $0.35 \%$ C8E4. TtOmp85 bound not to denatured matrix-bound MalK but to TtoA containing the signature sequence, while it bound only weakly to the mutant missing this motif (Fig. 8). Our results show that TtoA binds with its Cterminus to TtOmp85, giving a first hint that TtOmp85 plays a role in the assembly of TtoA.

\section{Discussion}

In this study, we identified a new $\beta$-barrel OMP of T. thermophilus HB27 and solved its structure, representing the first structure of an OMP from a thermophilic bacterium. TtoA was found in a bioinformatic screen searching for secreted $\beta$ strand-rich proteins containing a C-terminal signature sequence. This sequence should correspond to the last $\beta$-strand of the barrel in OMPs from Gramnegative bacteria, ${ }^{15}$ and this was confirmed in the case of TtoA. Apart from TtoA, only two other OMPs were identified in $T$. thermophilus HB27: TtOmp85 and TTC1475, a putative OMP. However, our screen was not designed to identify OMPs with an N-terminal barrel and a C-terminal periplasmic domain as known to exist in E. coli (e.g., OmpA). ${ }^{16}$ Thus, it is expected that there are further $\beta$-barrel OMPs in T. thermophilus HB27. Having characterized one $\beta$-barrel OMP, TtoA, we shall be able to inves- tigate its biogenesis in detail. In this work, we provide first hints that the OMP TtOmp85 is involved in the assembly of TtoA. In vitro, denatured TtoA bound directly to TtOmp85, but only weak interaction was observed in a TtoA mutant missing the signature sequence. This is in accordance with data available for E. coli. In planar lipid bilayer experiments, denatured OMPs also bound specifically to YaeT, an Omp85 family protein. ${ }^{15}$ In NMR experiments, peptides corresponding to internal $\beta$-strands of PhoE bound nonspecifically to the POTRA domains of YaeT, ${ }^{30}$ a behavior that might explain the weak residual binding of TtOmp85 to the Cterminal signature sequence mutant of TtoA.

TtoA is a major OMP of T. thermophilus HB27 as estimated from a Coomassie-stained polyacrylamide gel of separated isolated OMPs; however, the function of TtoA remains unknown. The structure suggests that TtoA is not a channel for small hydrophilic compounds: A barrier for hydrophilic compounds is located in the lower half of the $\beta$ barrel lumen, preventing a continuous channel. The barrel is connected to a hydrophobic funnel-shaped part on the extracellular side, making it also unlikely for hydrophilic compounds to pass. However, a possible entrance for hydrophilic substances could be the region where extracellular loops 1, 2 and 4 face one another and the head group of one detergent molecule is bound (Fig. 4). This region is positioned right above the upper girdle of aromatic side chains and thus located on the hydrophilic extracellular side of the membrane.

This raises the question as to the function of other known eight-stranded $\beta$-barrel OMPs. One of these, OmpW, has been proposed to be a transporter for small hydrophobic substances. ${ }^{26}$ The others have several different functions in the OMs of modern Gram-negative bacteria. PagP ${ }^{27}$ and $\mathrm{PagL}^{28}$ are enzymes that modify the lipid A portion of the lipopolysaccharide; PagP adds a palmitoyl moiety, whereas PagL removes an acyl chain. OmpA is involved in connecting the OM to the peptidoglycan, ${ }^{31}$ while $\mathrm{OmpX}^{25}$ and $\mathrm{NspA}^{29}$ are proposed to be involved in adhesion to host cells. In most eightstranded $\beta$-barrel OMPs, as in TtoA, the $\beta$-barrel might simply represent an economic and stable way to anchor a functional part to the OM, which in TtoA could be the funnel-like, large extracellular part. Searches for structural homologs with the DALI server did not yield proteins with similar parts, suggesting that its structure is unique. However, two features pointing to a possible function are shared with other eight-stranded $\beta$-barrel OMPs. The central hydrophobic patch is also present in OmpW, NspA and PagP. In the structure of these three proteins, a detergent molecule was bound in the hydrophobic patch. Although we did not observe any continuous and defined electron density in the structure of TtoA, it is possible that this patch serves as a binding pocket for hydrophobic compounds, since it seems too dominant and energetically disadvantageous to be permanently solvent exposed. The most prominent part of TtoA is the five-stranded extracellular $\beta$-sheet 
protruding out of the $\beta$-barrel. On the edge of the $\beta$ sheet, the exposed $\beta$-strand 10 forms crystal contacts with $\beta$-strand 10 of a neighboring molecule. The same behavior was observed for OmpX where $\beta$-strand 6 of a four-stranded $\beta$-sheet forms crystal contacts with $\beta$ strand 6 of the other protein. It was proposed that during infection, OmpX interacts with proteins containing a complementary $\beta$-strand at their surface, thereby promoting adhesion to host cells. We cannot speculate about possible interaction partners in hot springs, but it could be that TtoA interacts via this $\beta$ strand with the S-layer protein or extracellular proteins of the same or other cells. Such an interaction may be important for stabilization of the OM or functional reasons, for triggering a structural conversion required for substrate binding or release, for example.

A couple of factors are thought to contribute to the thermostability of soluble proteins from microorganisms living at high temperatures, ${ }^{32}$ but the thermostability of integral membrane proteins is less well investigated. We compared the structure of TtoA with the structures of the six eightstranded $\beta$-barrel OMPs of known structure from mesophiles since the structures of the TtoA homologs are not solved. There is no clear difference between the OMPs pointing to thermostability; therefore, the thermostabilization of the $\beta$-barrel part might depend more on interactions with the lipid bilayer, which is known to be adapted to high temperatures. 6,7

Thermus and Deinococcus spp. form a distinct eubacterial phylogenetic lineage, and they are believed to represent an intermediate between Gram-positive and Gram-negative bacteria. The unrelated mycobacteria represent another intermediate with an unusual OM. The structure of the OMP MspA of Mycobacterium smegmatis revealed a $\beta$-barrel structure that differs completely from that of modern Gram-negative bacteria. ${ }^{33}$ In contrast, the overall structure and the transmembrane part of TtoA fit very well into the structures of OMPs from modern Gram-negative bacteria. Our data also indicate that the biogenesis of TtoA via the Omp85 family protein TtOmp85 is evolutionarily conserved, making T. thermophilus an interesting organism with which to investigate the mechanism of insertion and assembly of OMPs into the OM in detail.

\section{Materials and Methods}

\section{Bacterial strains and growth conditions}

T. thermophilus strains HB27, HB8 (obtained from the Deutsche Sammlung von Mikroorganismen und Zellkulturen, Braunschweig, Germany), HB27: :nar pMKE2 TtOmp85 $5^{10}$ and HB8 pMK18-His-TTC0834 ${ }^{18}$ as well as E. coli strains Mach1-T1 and BL21-AI (Invitrogen) were used in this study. T. thermophilus was grown at $70{ }^{\circ} \mathrm{C}$ in medium containing $8 \mathrm{~g} / 1$ of trypticase, $4 \mathrm{~g} / 1$ of yeast extract and $3 \mathrm{~g} / \mathrm{l}$ of $\mathrm{NaCl}$ dissolved in $\mathrm{dH}_{2} \mathrm{O}$ (pH 7.5). E. coli was grown in LB medium at $30{ }^{\circ} \mathrm{C}$. Kanamycin was used at $25 \mu \mathrm{g} / \mathrm{ml}$ for E. coli and T. thermophilus.

\section{Plasmid construction}

DNA work was carried out using standard procedures. ${ }^{34}$ Chromosomal DNA from T. thermophilus was prepared as described recently for E. coli ${ }^{35}$ Phusion DNA Polymerase (Finnzymes) was used for PCRs. A DNA fragment coding for mature TtoA was amplified by PCR from chromosomal HB27 DNA using primer "for1" 5'GGCAGCCATATGCAGAAGTTCTCTGTAGAGGC3' (NdeI site underlined) and 5 'CTCGAGTGCGGCCGCTTAGAACCGATAGG CCGCC3' (NotI site underlined). Plasmid pET28-HisTtoA was obtained by digesting the PCR product with NdeI and NotI and ligating it into NdeI- and NotI-digested pET28a. Plasmid pET28-HisTtOmp85 was constructed similarly by using primers 5'GCAGCCATATGGCCCCCTTACGGGAGATCGT3' and 5'CTCGAGTGCGGCCGCTTAGAACATGGGCCCGATGCG3' for amplification of DNA encoding Omp85 without signal sequence. A DNA fragment coding for Tto $\mathrm{A}$ without the signal sequence and the last nine C-terminal residues (TtoA $\triangle 9 \mathrm{C}$ ) was amplified using primer "for1" and 5'GCGAATTCTTACTTGAGCACCCACTCGGGCT3' (EcoRI site underlined) and was ligated into pET28 digested with NdeI and EcoRI. Plasmid pET28-His-MalK was constructed by inserting malK between the NdeI and EcoRI sites of pET28a. malK was amplified from chromosomal HB27 DNA using primers $5^{\prime}$ CGGCAGCCATATGGCCAAGGTCAGGCTGGA3' and 5' TCGAATTCCTAGCGGGCCACGGCCGCCC3'. All inserts of the plasmids were sequenced (GATC, Konstanz).

\section{Protein purification under denaturing conditions}

His-Omp85, His-Tto A and His-TtoA $\triangle 9 \mathrm{C}$ were expressed as inclusion bodies in E. coli BL21-AI from plasmids pET28-HisTtOmp85, pET28-HisTtoA and pET28-HisTtoA $\triangle 9 \mathrm{C}$. At an optical density of 0.8 , cells grown at $30^{\circ} \mathrm{C}$ were induced for $14 \mathrm{~h}$ at $20{ }^{\circ} \mathrm{C}$ with $0.2 \%$ arabinose. Cell pellets were resuspended in phosphatebuffered saline (PBS) and lysed by passing it through a French pressure cell. Inclusion bodies were collected as a pellet by centrifugation at $6000 \mathrm{~g}$ for $20 \mathrm{~min}$ at $4{ }^{\circ} \mathrm{C}$. Inclusion bodies were solubilized in $20 \mathrm{mM}$ Na-phosphate, $500 \mathrm{mM} \mathrm{NaCl}, 20 \mathrm{mM}$ imidazole and $8 \mathrm{M}$ urea $\mathrm{pH} 7$, and insoluble material was removed by ultracentrifugation $\left(100,000 \mathrm{~g}, 1 \mathrm{~h}, 4^{\circ} \mathrm{C}\right)$. His-TtOmp85, His-TtoA and His-Tto $A \triangle 9 \mathrm{C}$ were purified further by Ni-affinity chromatography on a His-Trap column (Amersham) and dialyzed against PBS/8 $\mathrm{M}$ urea.

\section{Purification of native proteins}

T. thermophilus MalK was expressed with an N-terminal His tag in E. coli BL21-AI from plasmid pET28-His-MalK and purified by using Ni-affinity chromatography. Native TtOmp85 was purified from T. thermophilus HB27: :nar pMKE2-TtOmp85 cells as described previously. ${ }^{10}$ TtoA was purified together with TtOmp85; separation was achieved by gel-filtration chromatography. For crystallization, His-TtoA was expressed in T. thermophilus HB8 harboring plasmid pMK18-HisTTC0834, which expresses TtoA from its own promoter, with its signal sequence and a His tag followed by two Ala residues inserted after the cleavage site of the signal peptidase at position 2 of the mature sequence. TtoA was purified using Ni-affinity and gel-filtration chromatography as described recently. ${ }^{18}$ 


\section{OM preparations}

OMPs were isolated by sucrose gradient centrifugation as described previously. ${ }^{10}$ For the identification of proteins, OM preparations of strain HB27 were separated by SDS-PAGE ${ }^{36}$ and stained with Coomassie G250 (Serva). Protein bands were cut out and subjected to mass spectrometric analysis (Proteome Factory, Berlin, Germany).

\section{In vitro pull-down assay}

The bait proteins, purified denatured His-TtoA, denatured His-Tto $A \triangle 9 \mathrm{C}$ and His-MalK denatured in urea shortly before the assay, were bound to Ni-Sepharose beads (Amersham) in PBS containing $8 \mathrm{M}$ urea. The beads were then incubated with the prey protein, purified native TtOmp85 from HB27, in PBS containing 0.35\% C8E4 for $30 \mathrm{~min}$ at room temperature. The beads were washed five times with PBS containing $0.35 \%$ C8E4 and subsequently boiled for $10 \mathrm{~min}$ in SDS-PAGE loading buffer to get bound proteins for Western blot analysis.

\section{Western blot analysis}

Purified denatured His-TtOmp85, denatured His-TtoA and native His-MalK were used for immunization of rabbits at the TFA (Tierforschungsanstalt, University of Konstanz) to produce anti-TtOmp85, anti-TtoA and antiMalK antibody sera. Proteins were blotted onto a nitrocellulose membrane. Antirabbit immunoglobulin Galkaline phosphatase (Sigma) was used as the secondary antibody. Chemiluminescence detection of the phosphatase signal was performed using Immobilon Western AP Substrate (Millipore), and signals were recorded with an LAS3000 imager (Fujifilm).

\section{Crystallization and data collection}

Crystallization of native His-TtoA in $0.2 \mathrm{M}$ sodium malonate and $44 \%$ methylpentanediol, native data collection and determination of space group $P 3_{1} 21$ were done as described previously. ${ }^{18}$ To further improve crystal quality, we performed additional fine-screening experiments using hanging-drop vapor-diffusion setups. These crystals, grown in $0.2 \mathrm{M}$ sodium malonate and $43 \%$ methylpentanediol, showed the same space group and isomorphous unit cell parameters but increased resolution to $2.8 \AA$ (Table 1). The improvement in resolution should be mainly due to increased crystal size, as multiple drops over the same reservoir showed different crystallization kinetics, resulting in different numbers and sizes of crystals. Data sets of optimized crystals were collected at beamline PXI of the SLS (Swiss Light Source, Villigen, Switzerland). Another data set was collected at a wavelength of $1.77 \AA$ to identify potentially present anomalous signals.

\section{Heavy atom derivatization}

As attempts to solve the structure by molecular replacement using known structures of eight-stranded $\beta$-barrels were not successful, we determined experimental phases via heavy atom derivatization. Crystals of Tto ${ }^{18}$ were soaked in crystallization buffer supplemented with $10 \mathrm{mM} \mathrm{K}{ }_{2} \mathrm{PtCl}_{4}$ for periods varying between $20 \mathrm{~min}$ and $24 \mathrm{~h}$. Derivative crystals were back soaked in crystallization buffer without $\mathrm{K}_{2} \mathrm{PtCl}_{4}$ for at least $1 \mathrm{~min}$ prior to flash freezing in liquid nitrogen. No additional cryo-protection agent was added. The best data set was obtained with a derivative crystal of TtoA soaked for $90 \mathrm{~min}$ in $\mathrm{K}_{2} \mathrm{PtCl}_{4}$ that diffracted up to $3.5 \AA$ and remained stable during the whole data collection. Seven hundred twenty frames of $0.5^{\circ} \Delta \varphi$ were collected (beamline PXI at SLS). The data set was isomorphous to the native data sets and was used for determination of the phases via SIRAS.

\section{Phasing, structure solution, model building and refinement}

Data sets (Table 1) were processed using the program XDS. ${ }^{37}$ For initial structure solution, the native data set of $3.2 \AA^{18}$ and the data set of the $\mathrm{K}_{2} \mathrm{PtCl}_{4}$ derivative, with cutoff at $3.5 \AA$ resolution, were used. Heavy atom sites were identified using the program ShelxD, ${ }^{38}$ the coordinates of heavy atoms sites were refined and phases were obtained through SIRAS using the program SHARP/ AutoSHARP. ${ }^{39}$ The resulting experimental map showed three monomers of TtoA per asymmetric unit. The quality of the electron density differed slightly between the monomers, with the main differences in intracellular and extracellular loop regions. The best defined monomer was used for initial model building according to the amino acid sequence of TtoA. This model was used to place two additional monomers into the asymmetric unit by molecular replacement using the program Molrep ${ }^{40}$ as implemented in CCP4. ${ }^{41}$ Subsequently, chains A, B and C were refined and improved independently. Two TLS (translation/libration/screw) groups ${ }^{42,43}$ were defined for each monomer, but no NCS (noncrystallographic symmetry) restraint was applied. Refinement was done with the program Phenix.refine. ${ }^{44}$ During subsequent steps of model building and refinement, the data to 2.8 $\AA$ resolution became available. The same $R_{\text {free }}$ set as that for the $3.2 \AA$ resolution data was applied to the improved data set and extended up to $2.8 \AA$ resolution to obtain coherent $R_{\text {free }}$ values. Model building was done with the program Coot. ${ }^{45}$

Two hundred seven residues of monomer A could be built. However, due to different conformations and extended discontinuities in the electron densities for the loop region between residues 86 and 89 of monomers $B$ and $C$, these segments are missing in the final model of chains B and C. Refinement statistics are given in Table 1 .

\section{Bioinformatic analysis and figure preparation}

All annotated proteins from T. thermophilus HB27 were downloaded from TIGR $\dagger$. A customized program was written to identify proteins containing the C-terminal recognition site $(\mathrm{H}-\mathrm{X}-\mathrm{H}-\mathrm{X}-\mathrm{H}-\mathrm{X}-\mathrm{H} / \mathrm{Y}-\mathrm{X}-\mathrm{W} / \mathrm{F}$, where " $H$ " is a hydrophobic residue and " $X$ " is any residue). The identified proteins were analyzed for an $\mathrm{N}$-terminal signal sequence using SignalP $3.0^{46}$ and for the presence of $\beta$-strands using PRED-TMBB. ${ }^{47}$ Blast $^{48}$ search was performed to identify homologs of TtoA, and the obtained sequences were aligned with Kalign. ${ }^{49}$ Structural alignments with other known structures of eight-stranded $\beta$ barrels were done with the DALI server:.50 The server was also used to search for similar domains deposited in the Protein Data Bank with the extracellular part of TtoA as a

$\dagger$ http://www.jcvi.org

thttp://ekhidna.biocenter.helsinki.fi/dali_server/ 
template. The program DSSP was used to assign secondary structure elements. ${ }^{51}$ The topology figure of TtoA was drawn with TopDraw, ${ }^{52}$ and all figures of the atomic model of TtoA were prepared with PyMOL. ${ }^{53}$

\section{Accession numbers}

The coordinates and structure factors have been deposited in the Protein Data Bank§ with ID code 3dzm.

\section{Acknowledgements}

This work was supported by the Deutsche Forschungsgemeinschaft through grant WE962/11-1. We are grateful to Stefanie Krieg and Karsten Schäfer for their helpful input during structure solution. We thank the staff of the synchrotron beamline PXI at the SLS for their technical assistance and beamline support. We also thank Anne Keller for carefully reading the manuscript.

\section{References}

1. Gupta, R. S. (2000). The phylogeny of proteobacteria: relationships to other eubacterial phyla and eukaryotes. FEMS Microbiol. Rev. 24, 367-402.

2. Quintela, J. C., Pittenauer, E., Allmaier, G., Aran, V. \& de Pedro, M. A. (1995). Structure of peptidoglycan from Thermus thermophilus HB8. J. Bacteriol. 177, 4947-4962.

3. Cava, F., de Pedro, M. A., Schwarz, H., Henne, A. \& Berenguer, J. (2004). Binding to pyruvylated compounds as an ancestral mechanism to anchor the outer envelope in primitive bacteria. Mol. Microbiol. 52, 677-690.

4. Caston, J. R., Berenguer, J., de Pedro, M. A. \& Carrascosa, J. L. (1993). S-layer protein from Thermus thermophilus HB8 assembles into porin-like structures. Mol. Microbiol. 9, 65-75.

5. Engelhardt, H. \& Peters, J. (1998). Structural research on surface layers: a focus on stability, surface layer homology domains, and surface layer-cell wall interactions. J. Struct. Biol. 124, 276-302.

6. Pask-Hughes, R. A. \& Shaw, N. (1982). Glycolipids from some extreme thermophilic bacteria belonging to the genus Thermus. I. Bacteriol. 149, 54-58.

7. Leone, S., Molinaro, A., Lindner, B., Romano, I., Nicolaus, B., Parrilli, M. et al. (2006). The structures of glycolipids isolated from the highly thermophilic bacterium Thermus thermophilus Samu-SA1. Glycobiology, $16,766-775$.

$\S$ http://www.pdb.org
8. Maier, E., Polleichtner, G., Boeck, B., Schinzel, R. \& Benz, R. (2001). Identification of the outer membrane porin of Thermus thermophilus HB8: the channelforming complex has an unusually high molecular mass and an extremely large single-channel conductance. J. Bacteriol. 183, 800-803.

9. Rumszauer, J., Schwarzenlander, C. \& Averhoff, B. (2006). Identification, subcellular localization and functional interactions of PilMNOWQ and PilA4 involved in transformation competency and pilus biogenesis in the thermophilic bacterium Thermus thermophilus HB27. FEBS J. 273, 3261-3272.

10. Nesper, J., Brosig, A., Ringler, P., Patel, G. J., Muller, S. A., Kleinschmidt, J. H. et al. (2008). TtOmp85 from Thermus thermophilus HB27: an ancestral type of the Omp85 protein family. J. Bacteriol. 190, 4568-4575.

11. Henne, A., Bruggemann, H., Raasch, C., Wiezer, A., Hartsch, T., Liesegang, H. et al. (2004). The genome sequence of the extreme thermophile Thermus thermophilus. Nat. Biotechnol. 22, 547-553.

12. Bruggemann, H. \& Chen, C. (2006). Comparative genomics of Thermus thermophilus: plasticity of the megaplasmid and its contribution to a thermophilic lifestyle. J. Biotechnol. 124, 654-661.

13. Schulz, G. E. (2002). The structure of bacterial outer membrane proteins. Biochim. Biophys. Acta, 1565, 308-317.

14. Tsukazaki, T., Mori, H., Fukai, S., Ishitani, R., Mori, T., Dohmae, N. et al. (2008). Conformational transition of Sec machinery inferred from bacterial SecYE structures. Nature, 455, 988-991.

15. Robert, V., Volokhina, E. B., Senf, F., Bos, M. P., Van Gelder, P. \& Tommassen, J. (2006). Assembly factor Omp85 recognizes its outer membrane protein substrates by a species-specific C-terminal motif. PLoS Biol. 4, e377.

16. Bos, M. P., Robert, V. \& Tommassen, J. (2007). Biogenesis of the Gram-negative bacterial outer membrane. Annu. Rev. Microbiol. 61, 191-214.

17. Rosenbusch, J. P. (1974). Characterization of the major envelope protein from Escherichia coli. Regular arrangement on the peptidoglycan and unusual dodecyl sulfate binding. J. Biol. Chem. 249, 8019-8029.

18. Brosig, A., Nesper, J., Welte, W. \& Diederichs, K. (2008). Expression, crystallization and preliminary $\mathrm{X}$-ray analysis of an outer membrane protein from Thermus thermophilus HB27. Acta Crystallogr., Sect. F: Struct. Biol. Cryst. Commun. 64, 533-536.

19. Diederichs, K. \& Karplus, P. A. (1997). Improved $R$-factors for diffraction data analysis in macromolecular crystallography. Nat. Struct. Biol. 4, 269-275.

20. Weiss, M. S., Abele, U., Weckesser, J., Welte, W., Schiltz, E. \& Schulz, G. E. (1991). Molecular architecture and electrostatic properties of a bacterial porin. Science, 254, 1627-1630.

21. Dokmanic, I., Sikic, M. \& Tomic, S. (2008). Metals in proteins: correlation between the metal-ion type, coordination number and the amino-acid residues involved in the coordination. Acta Crystallogr., Sect. D: Biol. Crystallogr. 64, 257-263.

22. Berenguer, J., Faraldo, M. L. \& de Pedro, M. A. (1988). $\mathrm{Ca}^{2+}$-stabilized oligomeric protein complexes are major components of the cell envelope of "Thermus thermophilus" HB8. J. Bacteriol. 170, 2441-2447.

23. Dutzler, R., Rummel, G., Alberti, S., Hernandez-Alles, S., Phale, P., Rosenbusch, J. et al. (1999). Crystal structure and functional characterization of OmpK36, the osmoporin of Klebsiella pneumoniae. Structure, 7, 425-434. 
24. Pautsch, A. \& Schulz, G. E. (1998). Structure of the outer membrane protein A transmembrane domain. Nat. Struct. Biol. 5, 1013-1017.

25. Vogt, J. \& Schulz, G. E. (1999). The structure of the outer membrane protein OmpX from Escherichia coli reveals possible mechanisms of virulence. Structure, 7, 1301-1309.

26. Hong, H., Patel, D. R., Tamm, L. K. \& van den Berg, B. (2006). The outer membrane protein OmpW forms an eight-stranded beta-barrel with a hydrophobic channel. J. Biol. Chem. 281, 7568-7577.

27. Ahn, V. E., Lo, E. I., Engel, C. K., Chen, L., Hwang, P. M., Kay, L. E. et al. (2004). A hydrocarbon ruler measures palmitate in the enzymatic acylation of endotoxin. EMBO J. 23, 2931-2941.

28. Rutten, L., Geurtsen, J., Lambert, W., Smolenaers, J. J., Bonvin, A. M., de Haan, A. et al. (2006). Crystal structure and catalytic mechanism of the LPS 3-Odeacylase PagL from Pseudomonas aeruginosa. Proc. Natl Acad. Sci. USA, 103, 7071-7076.

29. Vandeputte-Rutten, L., Bos, M. P., Tommassen, J. \& Gros, P. (2003). Crystal structure of Neisserial surface protein A (NspA), a conserved outer membrane protein with vaccine potential. J. Biol. Chem. 278, 24825-24830.

30. Knowles, T. J., Jeeves, M., Bobat, S., Dancea, F., McClelland, D., Palmer, T. et al. (2008). Fold and function of polypeptide transport-associated domains responsible for delivering unfolded proteins to membranes. Mol. Microbiol. 68, 1216-1227.

31. Koebnik, R. (1995). Proposal for a peptidoglycanassociating alpha-helical motif in the C-terminal regions of some bacterial cell-surface proteins. Mol. Microbiol. 16, 1269-1270.

32. Kumar, S., Tsai, C. J. \& Nussinov, R. (2000). Factors enhancing protein thermostability. Protein Eng. 13, 179-191.

33. Faller, M., Niederweis, M. \& Schulz, G. E. (2004). The structure of a mycobacterial outer-membrane channel. Science, 303, 1189-1192.

34. Sambrook, J., Fritsch, E. F. \& Maniatis, T. (1989). Molecular Cloning: A Laboratory Manual. Cold Spring Harbor Laboratory, Cold Spring Harbor, NY.

35. Grimberg, J., Maguire, S. \& Belluscio, L. (1989). A simple method for the preparation of plasmid and chromosomal E. coli DNA. Nucleic Acids Res. 17, 8893.

36. Laemmli, U. K. (1970). Cleavage of structural proteins during the assembly of the head of bacteriophage T4. Nature, 227, 680-685.

37. Kabsch, W. (1993). Automatic processing of rotation diffraction data from crystals of initially unknown symmetry cell constants. J. Appl. Crystallogr. 26, 795-800.

38. Schneider, T. R. \& Sheldrick, G. M. (2002). Substructure solution with SHELXD. Acta Crystallogr., Sect. D: Biol. Crystallogr. 58, 1772-1779.
39. de la Fortelle, E. \& Bricogne, G. (1997). Maximumlikelihood heavy atom parameter refinement for multiple isomorphous replacement and multiwavelength anomalous diffraction methods. Methods Enzymol. 276, 472-494.

40. Vagin, A. \& Teplyakov (1997). MOLREP: an automated program for molecular replacement. J. Appl. Crystallogr. 30, 1022-1025.

41. Collaborative Computational Project No. 4. (1994) The CCP4 suite: programs for protein crystallography. Acta Crystallogr., Sect. D: Biol. Crystallogr. 50, 760-763.

42. Painter, J. \& Merritt, E. A. (2006). Optimal description of a protein structure in terms of multiple groups undergoing TLS motion. Acta Crystallogr., Sect. D: Biol. Crystallogr. 62, 439-450.

43. Painter, J. \& Merritt, E. A. (2006). TLSMD Web server for the generation of multi-group TLS models. J. Appl. Crystallogr. 39, 109-111.

44. Adams, P. D., Grosse-Kunstleve, R. W., Hung, L. W. Ioerger, T. R., McCoy, A. J., Moriarty, N. W. et al. (2002). PHENIX: building new software for automated crystallographic structure determination. Acta Crystallogr., Sect. D: Biol. Crystallogr. 58, 1948-1954

45. Emsley, P. \& Cowtan, K. (2004). Coot: model-building tools for molecular graphics. Acta Crystallogr., Sect. D: Biol. Crystallogr. 60, 2126-2132.

46. Bendtsen, J., Nielsen, H., von Heijne, G. \& Brunak, S. (2004). Improved prediction of signal peptides: SignalP 3.0. J. Mol. Biol. 16, 783-795.

47. Bagos, P. G., Liakopoulos, T. D., Spyropoulos, I. C \& Hamodrakas, S. J. (2004). PRED-TMBB: a Web server for predicting the topology of beta-barrel outer membrane proteins. Nucleic Acids Res. 32, W400-W404.

48. Altschul, S. F., Madden, T. L., Schaffer, A. A., Zhang, J., Zhang, Z., Miller, W. \& Lipman, D. J. (1997). Gapped BLAST and PSI-BLAST: a new generation of protein database search programs. Nucleic Acids Res. 25, 3389-3402.

49. Lassmann, T. \& Sonnhammer, E. L. (2006). Kalign, Kalignvu and Mumsa: Web servers for multiple sequence alignment. Nucleic Acids Res. 34, W596-W599.

50. Holm, L. \& Sander, C. (1996). Mapping the protein universe. Science, 273, 595-603.

51. Kabsch, W. \& Sander, C. (1983). Dictionary of protein secondary structure: pattern recognition of hydrogenbonded and geometrical features. Biopolymers, 22, 2577-2637.

52. Bond, C. S. (2003). TopDraw: a sketchpad for protein structure topology cartoons. Bioinformatics, 19, 311-312.

53. DeLano, W. L. (2002). The PyMOL Molecular Graphics System. www.pymol.org. 